\title{
Empleo de un agente de voladura de baja velocidad de detonación VOD (velocity of detonation) en voladura de carbón antracita
}

\author{
Use the blasting agent of low velocity of detonation VOD in blasting \\ anthracite coal
}

\author{
Ricardo Castillejo Melgarejo ${ }^{1}$ y Julio Poterico Huamayalli ${ }^{1}$
}

\section{RESUMEN}

La presente investigación está orientada a introducir en el proceso de minado el uso de un agente de voladura de baja velocidad de detonación VOD (velocity of detonation) con la finalidad de mejorar los parámetros de voladura en la producción de carbón del tipo antracita, el cual trasciende sustancialmente en la reducción de costos de la operación unitaria de perforación y voladura. Se presenta el procesamiento y análisis de los datos utilizando el análisis antes y después del empleo del agente de voladura, cuyos indicadores son presión en el taladro, fragmentación y costos; para determinar si existen diferencias se utilizó la prueba de T-Student, con un nivel de significación del 5\%. Se concluye que es factible el uso de los agentes de voladura de baja velocidad de detonación porque mejora de manera importante la fragmentación y reduce los costos de minado.

Palabras clave: Agente de voladura; fragmentación del carbón; costo.

\begin{abstract}
The research allows to introduce into the mining process is the use of a blasting agent low velocity of detonation VOD (velocity of detonation) with the purpose improve the blasting parameters in the production of anthracite coal and hence reduce unitary costs drilling and blasting. It presents detail the processing and analysis of data using analysis before and after the use agent blasting, whose indicators are borehole pressure, fragmentation and costs; to determinate if there are differences the statistical prove t-student was used, with a significance level 5\%. Finally, we present the results about the blasting parameters, leading to conclude that it is feasible to use a blasting agent that substantially improves fragmentation and reduce mining costs.
\end{abstract}

Keywords: Blasting agent; coal fragmentation; cost.

1 Universidad Nacional «Santiago Antúnez de Mayolo».Huaraz, Perú. 


\section{ICHIKLLACHAW}

Kay musyapakuywanqa imanaw mina kanqan riqitam qallaykuntsik, kaywanqa nakayllapa wip pashtaq kanqantam (VOD nishqan), kaywanqa Antrasita nishqan kaq laaya karwunpa pashtaynin alliyaananpaqraykurninmi, hurqayninchaw kaywanqa uchkuynin pashtaynin chaninpis uranmi. Kaychawqa llapan ruraynintam alliq churarayaamuu, ñawpa kaqwan qipa kaq pashtaypa ruraynintam, kayqa taladrupa prisyunnin, rakiynin, chaninkuna kanqantam riqitsimantsik; kaywan huklaayayninta riqipaq chanintsay istadistika t-student nishqantam wanakashqa, 5\% kanqan kaynintam. Ushayninmanqa, pashtaqapa chanintsaynin aykaman chanqantam churarayaamuu, kay llapan nishqantsikwanqa allim naqa wipyay pashtayta wanashwan, kayqa minadupa chanintam uchuyaatsinpis hinaman alliyaatsinpis.

Pushaq shimikuna: Pashtaynin; karwunpa uchukyaynin; chanin.

\section{INTRODUCCIÓN}

La presente investigación da cuenta sobre los parámetros de voladura con el empleo de los agentes de baja velocidad de detonación VOD (velocity of detonation) desarrollados en la mina Fernando Antonio F, y tiene como objetivo proponer a las distintas empresas de la región masificar el uso por capacidad de fragmentar el carbón, seguridad en su manipuleo y reducir los costos del proceso productivo.

En Áncash, se cuenta con yacimientos muy importantes del carbón del tipo antracita, en Yungay, Caraz, Huallanca, Sihuas, Yanama, Huallanca, Chavín, etc. La característica fundamental del carbón del tipo antracita es su elevado porcentaje de carbono y capacidad calorífica elevada; en este tipo de yacimiento sin genéticos, el contenido del gas «Grisú» no se registra y menos el metano, factores determinantes para el empleo de la técnica de perforación y voladura.

La coyuntura que actualmente muestra el mercado internacional, es que la demanda energética y siderúrgica está en incremento. Esto motiva que los productores de este combustible fósil incrementen su capacidad operativa y reduzcan los costos de minado; por tratarse de mantos de potencias superiores a los tres metros ( $3 \mathrm{~m}$ ), y buzamientos bastante variados, existen estratos de pequeña potencia de roca metamórfica «Lutita Pizarrosa» que en el proceso de voladura se tiene que mezclar con el carbón. En la región se comercializan tres productos, carbón duro destinado para la fabricación de los aceros, carbón grano como combustible para la producción de óxido de calcio y carbón cisco para las termoeléctricas o cementeras. Al usar un explosivo de alto VOD (velocity of detonation) se fragmenta al carbón en partículas muy finas, reduciendo su valor comercial sustancialmente por la mezcla entre la pizarra y el carbón. Lo que 
pretendemos con el empleo de los agentes de voladura es incrementar el tamaño de bloques de carbón y esto se logra con baja presión de explosión del taladro, lo cual implica hacer uso de agentes de bajo VOD (velocity of detonation).

El Ministerio de Energía y Minas, «Reglamento de seguridad y salud ocupacional» en el subcapítulo $\mathrm{V}$, agentes de voladura, en su artículo $255^{\circ}$ prohíbe el uso de los agentes de voladura en la explotación del carbón, criterio que no reúne ningún sustento científico, los yacimientos de carbón del departamento de Áncash son del tipo antracita dura, en el cual no hay la presencia del gas metano componente fundamental del gas «GRISU».

Los explosivos representados por las dinamitas, en formulación como agente sensibilizador lo compone la nitroglicerina, cuyo calor de formación supera ampliamente al ANFO para los diámetros de taladro que se maneja en las operaciones subterráneas. El DS-2010-EM, no prohíbe el uso de explosivos, que contradice a la teoría del poder calorífico entre el agente de voladura y el explosivo.

Los siniestros producidos en los yacimientos de carbón ocurren básicamente por la desgasificación del metano de los estratos del carbón en yacimientos del tipo bituminoso en que se registra porcentajes elevados del gas «GRISU».

Cadoni, Dotta (2010) sostiene que la impedancia acústica es una de las variables para seleccionar los explosivos para su mejor aprovechamiento en el proceso de fragmentación.

Scherpanisse, Silva (2010) concluye que el uso de los agentes con baja densidad reduce los costos, lo cual fue desarrollado en la universidad de Queens y probado en la mina Chuquicamata.

Cameron Mckenzie (2010), fundamenta que la presión del taladro está en función de la velocidad de detonación del agente de voladura y la densidad, de lo cual se concluye que las dinamitas para los diámetros de $38 \mathrm{~mm}$ tienen mayor presión en el taladro que el agente de voladura ANFO.

\section{MATERIALES Y MÉTODOS}

Para el estudio y evaluación de los parámetros de voladura se realizó la recopilación de datos mediante la información que se cuenta por cada disparo efectuado en las galerías del nivel Cecilia e Iniesta.

La aplicación de esta metodología nos permitió realizar un análisis cuantitativo y cualitativo sobre los parámetros de voladura teniendo como parámetro fundamental de granulometría del escombro. La presente investigación es descriptiva correlacional, 
descriptiva porque describe cómo la presión del taladro afecta la granulometría del escombro, correlacional por que mide el grado de relación que existe entre el antes y después, es decir, con el uso de explosivos y posteriormente con el uso de un agente de voladura.

Fuentes Primarias:

- Informes de producción

- Informes de productividad

Fuentes Secundarias:

- Análisis granulométrico del escombro

\section{RESULTADOS}

Tabla 1. Parámetros del diseño de perforación y voladura usando dinamita 65\%

\begin{tabular}{lc}
\hline PARÁMETRO DE VOLADURA & CANTIDAD \\
\hline $\mathrm{N}^{\circ}$ de Taladros & 21 \\
Diámetro del taladro & $36 \mathrm{~mm}$ \\
Longitud del taladro & $1.8 \mathrm{~m}$ \\
Longitud de carga & $1.2 \mathrm{~m}$ \\
Taco & Vacío \\
Explosivo & Dinamita $65 \%$ \\
Densidad & 1.14 \\
VOD & $5400 \mathrm{~m} / \mathrm{s}$ \\
Iniciador & Dinamita $65 \%$ \\
Ubicación del Iniciador & Fondo \\
Presión del taladro & $2092.50 \mathrm{Mpa}$ \\
Burdem & $0.7 \mathrm{~m}$ \\
Espaciamiento & $0.8 \mathrm{~m}$ \\
Perforación & Paralelo \\
Costo del disparo S/ & 36.95 \\
\hline
\end{tabular}


La tabla 1, muestra los parámetros de voladura haciendo uso como explosivo de la dinamita semigelatina de 65\%; muestra la presión que genera la detonación del taladro y el respectivo costo por disparo.

Tabla 2. Distribución granulométrica usando dinamita de 65\%

\begin{tabular}{cc}
\hline Diámetro pulg & \% Retenido \\
\hline $22-24$ & 0 \\
$20-22$ & 0 \\
$18-20$ & 1.3 \\
$16-18$ & 2.2 \\
$14-16$ & 2.1 \\
$12-14$ & 1.4 \\
$10-12$ & 1.1 \\
$8-10$ & 4.5 \\
$6-8$ & 6.7 \\
$4-6$ & 5.8 \\
$2-4$ & 14.8 \\
$1-2$ & 11.1 \\
$1 / 2-1$ & 24 \\
$1 / 8-1 / 2$ & 25 \\
$-1 / 8$ &
\end{tabular}

En la tabla 2 se aprecia la distribución granulométrica del escombro en la que se genera $60 \%$ como material fino y $3.5 \%$ como material grueso, lo cual es resultado del uso como explosivo de la dinamita semigelatina de $65 \%$.

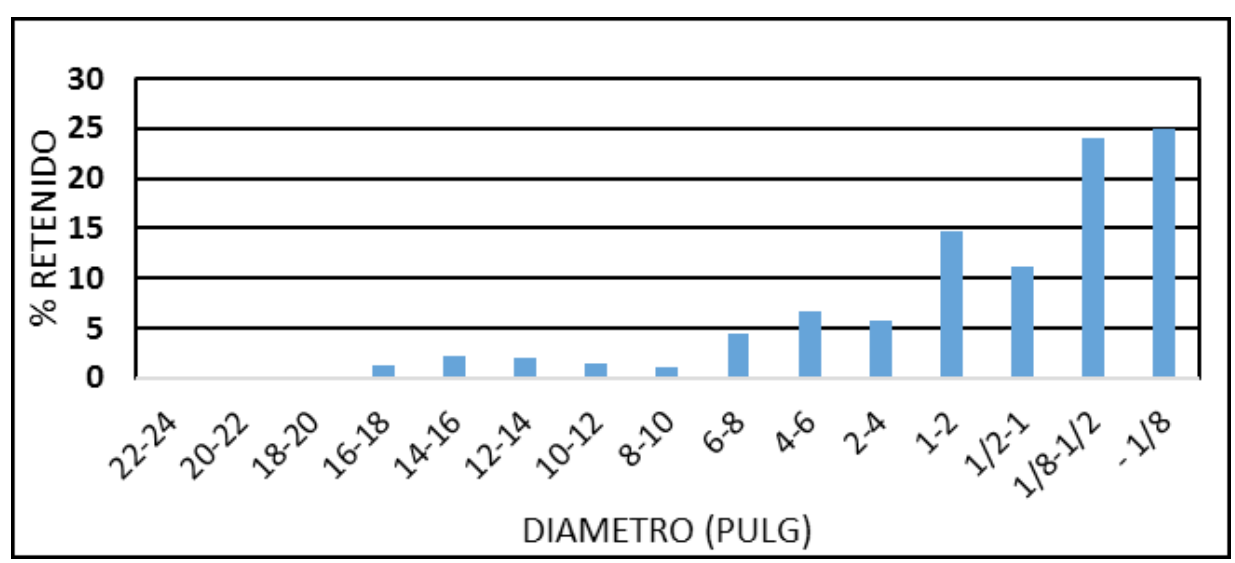

Figura 1. Distribución granulométrica - dinamita de 65\% 
La figura 1 muestra la distribución granulométrica por malla, observándose que el porcentaje mayor de los productos es material fino, es decir, de 1/8" a 1", el porcentaje de material grueso es mínimo.

Tabla 3. Parámetros del diseño de perforación y voladura usando ANFO

(Ammoniun Nitrate and Fuel Oil)

\begin{tabular}{lc}
\hline PARÁMETRO DE VOLADURA & CANTIDAD \\
\hline $\mathrm{N}^{\circ}$ de Taladros & 21 \\
Diámetro del taladro & $36 \mathrm{~mm}$ \\
Longitud del taladro & $1.80 \mathrm{~m}$ \\
Longitud de carga & $1.23 \mathrm{~m}$ \\
Taco & Vacío \\
Explosivo & ANFO (Superfram L) \\
Densidad & 0.56 \\
VOD & 2600 \\
Iniciador & Detonador $\# 8$ y dinamita $65 \%$ \\
Ubicación del Iniciador & Fondo \\
Presión del taladro & $247.69 \mathrm{Mpa}$ \\
Burdem & $0.7 \mathrm{~m}$ \\
Espaciamiento & $0.8 \mathrm{~m}$ \\
Perforación & Paralelo \\
Costo/disparo S/ & 21.23 \\
\hline
\end{tabular}

La tabla 3 muestra los parámetros de voladura haciendo uso del agente Superfram L, con VOD de $2600 \mathrm{~m} / \mathrm{s}$ que genera presión del taladro que es de 267.69 Mpa, y un costo de 21.23 soles por disparo. 
Tabla 4. Distribución granulométrica usando ANFO (Ammoniun Nitrate and Fuel Oil)

\begin{tabular}{cc}
\hline ø Pulg & \% Pasante \\
\hline $22-24$ & 0 \\
$20-22$ & 4.2 \\
$18-20$ & 5.3 \\
$16-18$ & 11.6 \\
$14-16$ & 4.6 \\
$12-14$ & 1.8 \\
$10-12$ & 8.9 \\
$8-10$ & 9.14 \\
$6-8$ & 7.1 \\
$4-6$ & 5.8 \\
$2-4$ & 3.7 \\
$1-2$ & 9.12 \\
$1 / 2-1$ & 5.7 \\
$1 / 8-1 / 2$ & 10.14 \\
$-1 / 8$ & 12.9 \\
\hline
\end{tabular}

La tabla 4 visualiza el resultado de la distribución granulométrica haciendo uso del ANFO como agente de voladura, en que se observa la reducción sustancial del porcentaje de finos en el escombro.

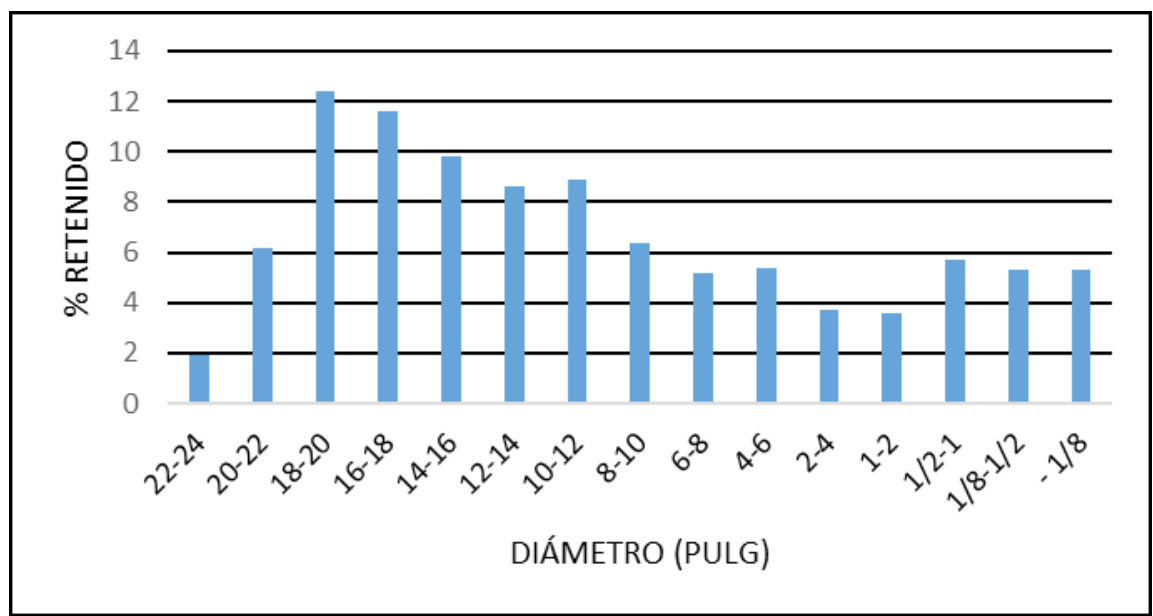

Figura 2. Distribución granulométrica usando ANFO (Ammoniun Nitrate and Fuel Oil) Fuente: Elaboración propia 
La figura 2 muestra gráficamente cómo el uso del agente de voladura de baja VOD reduce el porcentaje de material fino e incrementa el porcentaje de material grueso, lo cual favorece la reducción de costos e incremento del valor del carbón.

\section{DISCUSIÓN}

Los explosivos representados por la familia de las dinamitas poseen alto poder rompedor generando en el macizo rocoso un alto porcentaje de zona de trituración por las ondas expansivas. El volumen de gases que se desprende de la reacción química es mucho menor al del agente de voladura ANFO (Ammoniun nitrate and fuel oil). El costo unitario por kilogramo de explosivo es 13.13 soles, mientras que el agente de voladura es 3,35 soles por kilogramo. El ANFO (Ammoniun nitrate and fuel oil) es uno de los agentes de voladura más baratos en el mercado peruano. Su gran ventaja es la capacidad de empuje por alta generación de volúmenes de gases.

De acuerdo a lo señalado por Hustrulid (2102:206), Castilla (2013: 103), Berta (1985:83), la densidad de carga lineal de las dinamitas superan ampliamente a la densidad de carga del ANFO, por lo que se pudo evidenciar en la presente investigación que para taladros de $38 \mathrm{~mm}$ usando dinamitas se logra una densidad de carga lineal de 1.29 $\mathrm{kgr} / \mathrm{m}$ y para el agente de voladura se tiene la densidad de carga lineal de $0.97 \mathrm{kgr} / \mathrm{m}$ que favorece de manera notable la reducción de la presión en el taladro.

Scherpenisse (2010:117), sostiene que el uso de los agentes de voladura reducen sustancialmente los costos de minado, lo cual ratifica con los resultados obtenidos una reducción de 36.95 soles/disparo a 21.23 soles/disparo en el minado de carbón del tipo antracita en la mina Fernando Antonio F.

Las dinamitas al tener densidades superiores a $1.14 \mathrm{gr} / \mathrm{cc}$ y velocidades de detonación que supera a $5400 \mathrm{~m} / \mathrm{s}$, generan presiones superiores a $2092.50 \mathrm{Mpa}$ el cual ocasiona que en la zona de influencia del taladro se logre obtener mayor porcentaje de mineral muy fino; por la baja densidad del ANFO(Ammoniun nitrate and fuel oil) $0.86 \mathrm{gr} / \mathrm{cc}$ y la baja velocidad de detonación $2600 \mathrm{~m} / \mathrm{s}$ genera presión en el taladro de $247.67 \mathrm{Mpa}$ el cual permite obtener mayor porcentaje de mineral grueso que favorece de manera muy importante al productor minero, el cual contrasta con lo expuesto por Cameron Mckenzie (2010:96), quien señala que la presión del taladro haciendo uso de las dinamitas es mayor que el uso del agente de voladura ANFO para diámetros $38 \mathrm{~mm}$ que es de uso común en la minería convencional subterránea.

Cadoni (2010:35) plantea que la impedancia acústica es un parámetro fundamental para el apropiado aprovechamiento efectivo de un explosivo, por lo que la presente investigación demuestra que las rocas con baja impedancia acústica necesitan explosivos con baja densidad y baja velocidad de detonación. El carbón antracita con propiedades físicas de densidad de $1.25 \mathrm{t} / \mathrm{m} 3$ y velocidad sísmica de $1200 \mathrm{~m} / \mathrm{s}$ requiere para su fragmentación un agente de voladura de baja densidad y baja velocidad de detonación. 
El Ministerio de Energía de Minas (2010:76) en el reglamento de seguridad y salud ocupacional prohíbe el uso del ANFO en yacimientos de carbón, sin especificar si son del tipo bituminoso o antracita. Con los resultados del presente trabajo se concluye técnica y científicamente que no hay parámetros que restrinjan el uso del agente de Voladura de bajo VOD en yacimiento de carbón del tipo antracita.

El comercio de carbón está destinado básicamente al consumo local, como carbón siderúrgico y termoeléctrico. Dependiendo del mercado es conveniente producir en mayor porcentaje carbón de diámetros que superen a las 3 pulgadas para lograr mayor valor del carbón en el mercado.

Los resultados obtenidos en el rubro anterior nos conducen a sacar algunas conclusiones que serán dadas en forma resumida. Los costos por uso explosivo se han reducido en S/15.72 por disparo, el valor del mineral se ha incrementado en S/43.68, mejorando sustancialmente los ingresos de S/19947.50 a S/26500.00, en la minera San Roque FM SAC.

\section{CONCLUSIONES}

Las propiedades mecánicas del carbón antracita son bajas, con un valor promedio de 35.4 Mpa, mientras que la propiedad física de densidad es de $1.25 \mathrm{t} / \mathrm{m}^{3}$. Las características del macizo rocoso (carbón antracita), básicamente representado por RMR (rock mass raiting) está de 35 a 41, lo cual indica que es una roca de mala calidad. Los explosivos de alta velocidad de detonación logran fragmentar el carbón generando mayor porcentaje de material fino, mientras que el uso del ANFO (Ammoniun nitrate and fuel oil) incrementa de manera notable el porcentaje de material grueso y favorece la reducción del costo por disparo. Con el uso del agente de voladura de baja VOD (velocity of detonation) se incrementa sustancialmente el valor del mineral. Una de las propiedades del ANFO (Ammoniun nitrate and fuel oil) en el proceso de detonación es la generación de altos volúmenes de gases representados por el agua en vapor, dióxido de carbono y nitrógeno. Por lo tanto, sí es factible el uso del ANFO (Ammoniun nitrate and fuel oil) en los yacimientos de carbón del tipo antracita ubicados en el departamento de Áncash. Así mismo se concluye que se debe reformular el Decreto Supremo del 2010 publicado por el Ministerio de Energía y Minas «Reglamento de seguridad y salud ocupacional» en el cual se restringe el uso del ANFO en yacimientos de carbón del tipo antracita.

\section{REFERENCIAS BIBLIOGRÁFICAS}

Berta, Giorgio. 1985. L'esplosivo strumento di lavoro. Milano: Italesplosivi.

Castilla, Jorge. 2013. Perforación y voladura de rocas en minería. Madrid: Universidad Politécnica de Madrid. 
Cadoni, Ezio. 2010. Mecanical characterization of rocks at high strain. Londres: Edit. Taylor $\&$ francis group.

Hustrulid, Mark. 2012. Open pit mine planning and design. Colorado: Taylor \& Francis Group.

Mckenzie, Cameron. 2010. Tecnología de voladura de rocas. Colorado: Blastronic pty.

Ministerio de Energía y Minas. 2010. Reglamento de seguridad y salud ocupacional, Lima: MEM.

Scherpenisse, Carlos 2010. Development of low density reactive agents, Edit. Taylor \& francis group.

Fecha de recepción: 28 de enero 2016

Fecha de aceptación: 23 de mayo 2016

\section{Correspondencia}

Ricardo Castillejo Melgarejo

rcastillejo@techemail.com 